\title{
The impact of n-hexane on the secretion of mouse estrogen and progesterone
}

\author{
Huiling Huang ${ }^{1}$, Jin Liu' ${ }^{2}$ Fen Pang ${ }^{2}$, Wenchang Zhang ${ }^{2 *}$ \\ ${ }^{1}$ Union Hospital, Fujian Medical University, Fuzhou, China; \\ ${ }^{2}$ Department of Occupational and Environmental Health, University of Public Health, Fujian Medical University, Fuzhou, China. \\ Email: huanghuiling08@126.com, ljfast@sina.com, Pangfen0527@163.com, wenchang2008@126.com
}

Received 14 September 2011; revised 24 October 2011; accepted 18 November 2011.

\section{ABSTRACT}

Aim: To study the toxic effect of n-hexane on sex hormone levels, including its effect on ovarian sex hormone secretion levels and the underlying mechanism. 1) In vivo experiment: The mice were exposed to $n$ hexane by static inhalation of doses of $0,3.0,15.1$ or $75.8 \mathrm{ml} / \mathrm{m}^{3}$ once a day (4 hours), 7 days a week, for 5 consecutive weeks. After exposure, mouse sera were sampled to measure estradiol $\left(E_{2}\right)$ and progesterone $\left(P_{4}\right)$ levels by electrochemiluminescence immunoassays (ECLIA). 2) In vitro experiment: mice at different stages of the estrous cycle were sacrificed and their minced tissue pieces were incubated in medium containing hexane-2,5-dione (2,5-HD). Then, the $E_{2}$ and $P_{4}$ levels in the media were measured by ECLIA. In vivo assays, the serum $\mathrm{P}_{4}$ levels in each group were lower than the control group, which was statistically significant $(F=6.196, P<0.01)$. In the minced ovary tissue culture medium, the $E_{2}$ level of $75.8 \mathrm{~mL} / \mathrm{m}^{3}$ dosage group was decreased $(96.9522 \pm 7.8652))$ compared to the control group $(119.1600 \pm 25.6420)$, with statistical significance $(P<0.01)$. The $P_{4}$ levels of 15.1 $\mathrm{mL} / \mathrm{m}^{3}$ and $75.8 \mathrm{~mL} / \mathrm{m}^{3}$ dosage groups were also decreased $(18.5938 \pm 12.485 \mathrm{pmol} / \mathrm{L}, 13.5550 \pm 5.7789$ $\mathrm{pmol} / \mathrm{L})$ and were statistically significantly $(F=6.196$, $P<0.01)$ lower than that of the control group $(45.2213 \pm 16.5393 \mathrm{pmol} / \mathrm{L})$. In vitro, the $E_{2}$ levels at various stages of the estrous cycle were significantly different from the control groups $(P<0.05)$. Moreover, there was an interaction between the two variables $(P<0.05)$. In vitro, different concentrations of 2 , 5-HD significantly inhibited the $P_{4}$ level $(P<0.05)$. The $\mathbf{P}_{4}$ levels at various stages of the estrous cycle were also significantly different from controls $(P<$ $0.05)$, with no interaction $(P>0.05)$. The results indicate that $n$-hexane may inhibit release of ovarian $E_{2}$ and $P_{4}$, which may be the main reason for the interference of the reproductive endocrine system by $n$ hexane.
Keywords: n-Hexane; Sex Hormone; Interference of Reproductive Endocrine System

\section{INTRODUCTION}

$\mathrm{N}$-hexane is one of the five isomers of hexane and is an important industrial organic solvent that is widely used in the shoe and other industries. Many workers, especially female workers, are exposed to n-hexane. For example, in Fujian province alone, several thousands of female workers in shoe factories are exposed to n-hexane [1]. N-hexane is volatile and enters the body mainly as a gas through the respiratory tract under daily working conditions. N-hexane is mainly distributed in organs enriched in fatty acids, such as the brain, kidney, liver, spleen, testis and ovary [2]. Although the toxic mechanism of n-hexane on reproduction is still unclear, studies have shown that the active metabolic product of n-hexane is hexane-2, 5-Dione. There are many studies describing the neural toxicity of n-hexane, but fewer on the gonadal reproductive toxicity, especially on female gonadal reproductive toxicity. Recently, n-hexane interference of the female reproductive endocrine system has attracted much attention [3], and there are similar reports from China [4].

Ovarian sex hormone secretions play an important role in female gonadal reproductive function, such as estrus (menstrual status), the growth and development of follicle cells, as well as the levels of sex hormone levels in serum $[5,6]$. The current study investigated the impact of n-hexane on the secretion of mouse ovarian sex hormones using both in vivo and in vitro experiments. By comparing and analyzing the experimental results, the impact of n-hexane on the secretion of ovarian estrogen and progesterone and its possible mechanism were discussed.

\section{EXPERIMENTAL TECHNIQUES AND METHODS}

Reagents: n-hexane and hexane-2,5-dione (Shanghai Si- 
nopharm, AR grade), DMEM/F-12 medium (Hyclone), fetal bovine serum (Sigma), M2 medium (Sigma), human chorionic gonadotropin (HCG, Anhui Xinli Pharmaceutical Co., Ltd.)

Instruments: CK40-F200 BH-2 inverted microscope (Olympus, Japan), BH-200 microscope (Olympus, Japan), HF-safe 1200 biological safety cabinet (Shanghai Lishen Scientific Instrument Co. Ltd.), L-RDJ/1000 static inhalation chamber (Guangzhou JiuFang Electronics Co. Ltd.), Elecsys analyzer (Roche Diagnostics), constant temperature water bath shaker (Changzhou Guohua Electronics Co. Ltd.).

Experimental animals: 72 healthy adult female ICR mice, which were SPF with a body weight of $20-23 \mathrm{~g}$, were provided by Shanghai Slac Laboratory Animal Co. Ltd, Animal license No. SCXK (Hu) 2007-0005. The animal housing temperature was $20^{\circ} \mathrm{C} \pm 2^{\circ} \mathrm{C}$ and the humidity was $70 \% \pm 10 \%$. The plastic cages contained sterile sawdust bedding, and food and water were available ad libitum.

\subsection{Experimental Methods}

In vivo experiment: 40 female mice were randomly divided into 4 groups by stratified sampling according to their body weight (10 mice per group). The dosages of n-hexane were $0,3.0,15.1$ and $75.8 \mathrm{ml} / \mathrm{m}^{3}$. The uptake of n-hexane was by static inhalation for 4 hours a day, 7 days a week, for 5 consecutive weeks. After exposure, blood was drawn, and the blood samples were placed at $4^{\circ} \mathrm{C}$ for 3 hours and were then centrifuged at $3000 \mathrm{rpm}$ for $15 \mathrm{~min}$. The supernatant was transferred to a $1.5-\mathrm{mL}$ Eppendorf tube and stored at $-20^{\circ} \mathrm{C} . \mathrm{E}_{2}$ and $\mathrm{P}_{4}$ were measured using Elecsys according to the manufacturer's instructions. All the samples were measured at the same time to minimize errors.

Ten days after the initial exposure to n-hexane, pap smears were used to check the estrus of the mice. After the exposure was finished, when the animal entered the estrus, both ovaries were removed and put into a culture tube containing $1 \mathrm{~mL}$ incubation medium. The ovaries were minced with eye scissors, and then 5 IU of HCG was added to the tube. The mixture was incubated in an isothermal water bath shaker at $34^{\circ} \mathrm{C}$ for 3 hours followed by centrifugation at $1500 \mathrm{rpm}$ for $15 \mathrm{~min}$ at $4^{\circ} \mathrm{C}$. The supernatant was aspirated and used for measuring $E_{2}$ and $\mathrm{P}_{4}$ levels in the medium by ECLIA.

In vitro experiment: orthogonal experiments were designed using the orthogonal $\mathrm{L}_{16}\left(4^{5}\right)$ (Table 1). The factors and levels are as follows (each combination was repeated 3 times):

Thirty-two adult female ICR mice were randomly chosen based on the sixteen combinations by the orthogonal $\mathrm{L}_{16}\left(4^{5}\right)$ experimental design before the experiment. Es-
Table 1. Factors and levels considered in orthogonal experiment.

\begin{tabular}{ccccc}
\hline Factor & Level 1 & Level 2 & Level 3 & Level 4 \\
\hline 2,5-HD (mmol/L) & 0 & 20 & 40 & 60 \\
Mouse estrous cycle & proestrus & estrus & metestrus & diestrus \\
\hline
\end{tabular}

trous cycles were monitored with pap smears every 12 hours. When animals entered the expected estrous stage (such as estrus), the ovaries were removed and transferred to a 1.5-mL Eppendorf tube containing $1 \mathrm{~mL}$ culture medium. The ovaries were minced with eye scissors, and then $5 \mathrm{IU}$ of HCG and $30 \mu \mathrm{L}$ of different concentrations of 2, 5-HD were added to the tubes. The tubes were incubated in an isothermal water bath shaker at $34^{\circ} \mathrm{C}$ for 3 hours followed by centrifugation at $1500 \mathrm{rpm}$ for 15 $\min$ at $4^{\circ} \mathrm{C}$. The supernatant was used for measuring $\mathrm{E}_{2}$ and $\mathrm{P}_{4}$ levels by ECLIA.

\subsection{Statistical Analysis}

Most of reported values were expressed as mean \pm S.D. (standard deviation). One-way ANOVAs was employed to compare control groups and different doses of n-hexane. Figures $\mathbf{4}$ and $\mathbf{5}$ used with Univariate Analysis of Variance in Genear linear model. In all cases, $\mathrm{P}<0.05$ was considered to be significant, and $\mathrm{P}<0.01$ was considered to be very significant. SPSS version 13.0.

\section{RESULTS}

\subsection{Ovaries Exposed to n-Hexane in Vivo}

General appearance: Mice in each group all appeared depressed, although to different degrees. They displayed slowed motor activity, depilation, decreased food appetite, rhabdomyolysis and ulcers in the abdominal area. However, only one animal in the high dose group died during the experiments.

The change in the level of hormones in the serum is shown in Figures $\mathbf{1}$ and 2. The results (Figure 1) show that there was no significant difference in $E_{2}$ serum levels compared to control animals $(P>0.05)$. However, the results (Figure 2) also show that the $\mathrm{P}_{4}$ serum levels decreased significantly $(\mathrm{P}<0.01)$ in the $3.0,15.1$ and 75.8 $\mathrm{mL} / \mathrm{m}^{3}$ groups compared to the control.

The change in the level of $\mathrm{P}_{4}$ in the ovary culture medium is shown in Figure 3. The results (Figure 3(a)) show that the level of $\mathrm{P}_{4}$ decreased significantly ( $\mathrm{P}<$ 0.05 ) in the 15.1 and $75.8 \mathrm{~mL} / \mathrm{m}^{3}$ groups compared to the control. The results (Figure 3(b)) also show that the level of $E_{2}$ decreased significantly $(P<0.05)$ in the 75.8 $\mathrm{mL} / \mathrm{m}^{3}$ group compared to the control.

\subsection{Ovaries Exposed to 2, 5-HD in Vitro}

The effects of the different concentrations of 2,5-HD and 


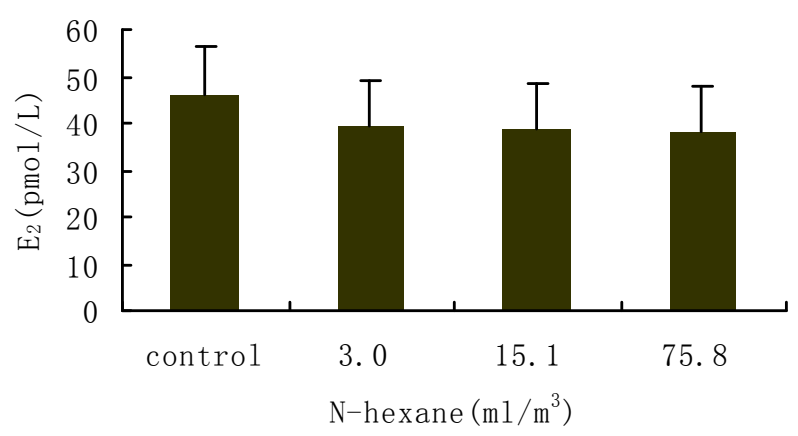

Figure 1. The level of serum $\mathrm{E}_{2}$ in the mouse treated with n-hexane (in vivo).

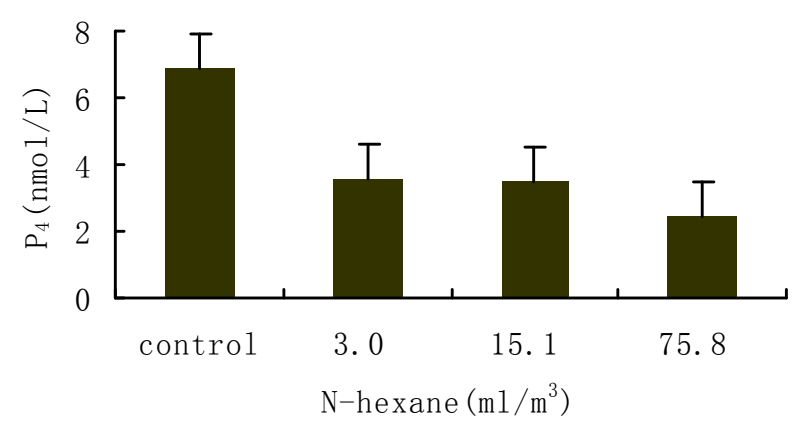

Figure 2. The level of serum $\mathrm{P}_{4}$ in the mouse treated with n-hexane (in vivo).

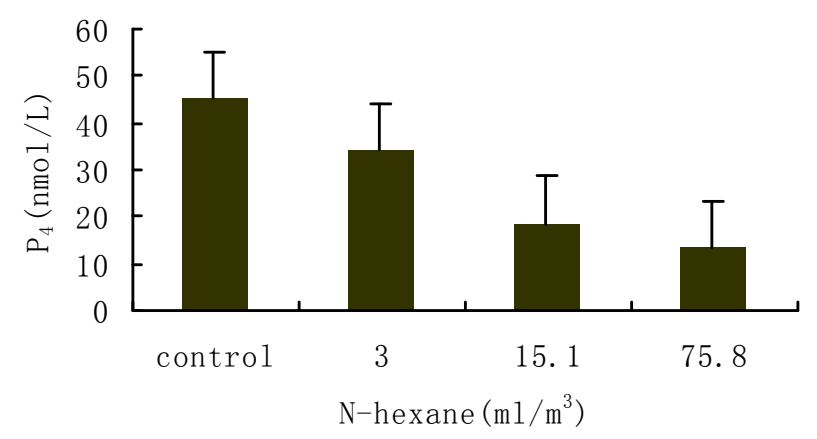

(a)

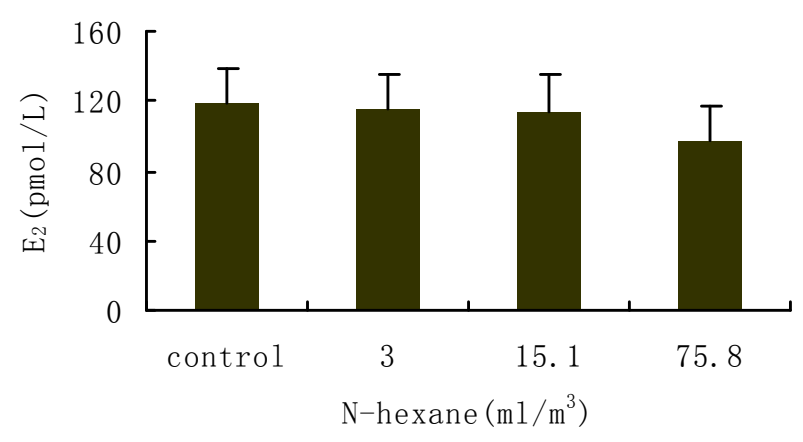

(b)

Figure 3. (a) The level of $\mathrm{P}_{4}$ in the culture serum in the ovaries treated with n-hexane (in vivo); (b) The level of $\mathrm{E}_{2}$ in the culture serum in the ovaries treated with n-Hexane (in vivo). different stages of estrous cycles on the level of $\mathrm{P}_{4}$ in the ovary culture medium are shown in Figure 4. The results show that the level of $\mathrm{P}_{4}$ in the ovaries (in the proestrus stage) declined $(\mathrm{P}<0.05)$ in the 40 and $60 \mathrm{mmol} / \mathrm{L}$ groups. Additionally, the level of $\mathrm{P}_{4}$ in the ovaries (in the estrus stage) decreased significantly $(\mathrm{P}<0.01)$ compared to the control. Moreover, the level of $\mathrm{P}_{4}$ in the ovaries (in the metestrus stage) decreased significantly $(\mathrm{P}<0.01)$ in the 40 and $60 \mathrm{mmol} / \mathrm{L}$ group compared to the control. Finally, the level of $\mathrm{P}_{4}$ in the ovaries (in the stage of diestrus) decreased significantly $(\mathrm{P}<0.01)$ in a dosedependent manner.

The effects of both different concentrations of 2, 5-HD and different stages of the estrous cycles on the level of $E_{2}$ in the culture serum in the ovaries are shown in Figure 4 . The results show that as the concentrations of 2,5-HD increased, the level of $\mathrm{E}_{2}$ in the ovaries (in the proestrus stage) declined $(\mathrm{P}<0.05)$ in the 40 and 60 $\mathrm{mmol} / \mathrm{L}$ groups. Additionally, the level of $\mathrm{E}_{2}$ in the ovaries (in the estrus stage) was not significantly different from the control. Moreover, as the concentrations of 2,5$\mathrm{HD}$ increased, the level of $\mathrm{E}_{2}$ in the ovaries (in the metestrus stage) declined $(\mathrm{P}<0.05)$ in the 40 and $60 \mathrm{mmol} / \mathrm{L}$ groups. The level of $\mathrm{E}$ in the ovaries (in the diestrus stage) decreased significantly $(\mathrm{P}<0.05)$ in the 40 and 60 $\mathrm{mmol} / \mathrm{L}$ groups compared to the control.

The effects of the different concentrations of 2, 5-HD and different stages of the estrous cycle on the level of $\mathrm{P}_{4}$ and $E_{2}$ in the culture serum of the ovaries are shown. The results (Figure 4) indicate that there were significant differences $(\mathrm{P}<0.05)$ in the level of $\mathrm{P}_{4}$ in the ovaries between the 20,40 , and $60 \mathrm{mmol} / \mathrm{L}$ groups compared to the control. There were also significant differences $(\mathrm{P}<0.05)$ in the level of $\mathrm{P}_{4}$ in the ovaries between different estrous cycles $(\mathrm{P}<0.05)$, but the interaction between them was not significant $(\mathrm{P}>0.05)$.

The results (Figure 5) show that there were significant differences $(\mathrm{P}<0.05)$ in the level of $\mathrm{E}_{2}$ in the ovaries between the 20, 40, and $60 \mathrm{mmol} / \mathrm{L}$ groups compared to the control. There were also significant differences $(\mathrm{P}<0.05)$ in the level of $E_{2}$ in the ovaries between estrus cycles (P $<0.05$ ), and the interaction between them was significant $(\mathrm{P}<0.05)$.

\section{DISCUSSION}

$\mathrm{N}$-hexane is a widely used industrial organic solvent, and many people have occupational exposure to it. In China for instance, in shoe manufacturers in Fujian, Guangdong, Zhejiang and other provinces, $n$-hexane is frequently used as a solvent for shoe glue in the factories. In the gluing process, n-hexane quickly evaporates into the air and enters human body. Therefore, the number of people who are exposed to $n$-hexane can be more than 10,000 , 


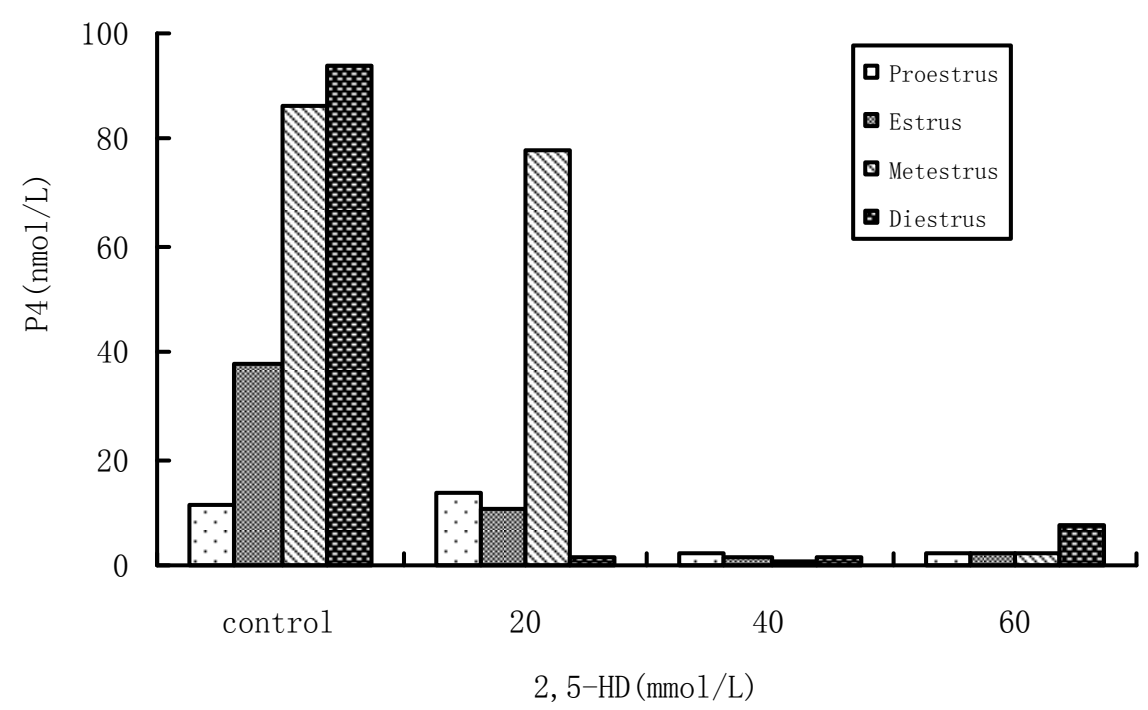

Figure 4. The level of $\mathrm{P}_{4}$ in the ovaries at different stages of the estrus cycle treated with 2, 5-HD (in vitro).

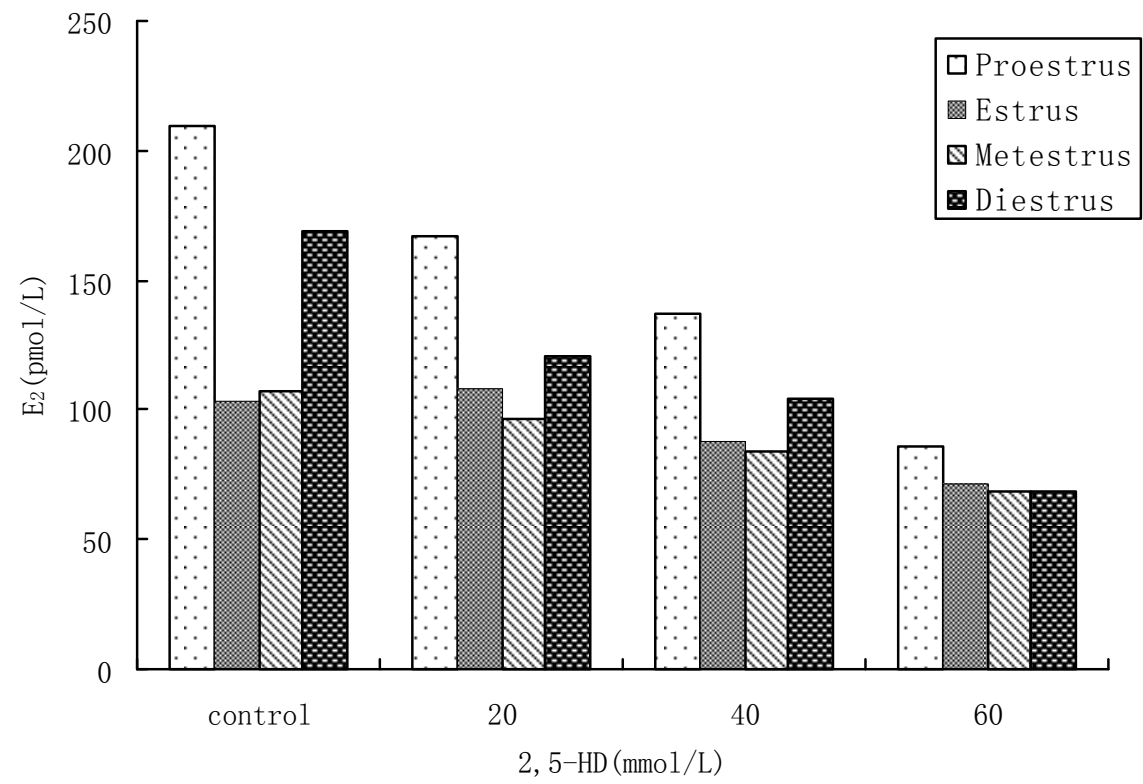

Figure 5. The level of $\mathrm{E}_{2}$ in the ovaries at different stages of the estrus cycle treated with 2, 5-HD (in vitro).

and more than $90 \%$ of them are female workers. Thus, it is very important to pay attention to the hazards of nhexane in regard to the health of female workers.

$\mathrm{N}$-hexane is a neurotropic toxin; studies in this field have already been systematically reported [7,8]. However, in recent years, the hazards of n-hexane on reproductive health, especially on female reproductive health, has become a great concern. Sallmen et al. conducted retrospective epidemiological research on 250 female workers in Portuguese shoe factories and 250 female workers in food stores and storehouses and analyzed FDR (fecundability density ratio) and its confidence interval (CI) value. They showed that organic solvents such as n-hexane and its isomers, toluene and methyl ketones, which are required in shoe manufacturing, damage the female reproductive system and result in reduced fertility [3]. There have been reports in China showing that nhexane can cause menopause and menstrual disorders in female patients [4]. However, studies in this field are still limited and the further damage caused by n-hexane and its possible mechanisms remain unclear. In our previous studies, we found that 2,5-HD caused significant apoptosis of human ovarian granulosa cells [9]. It is very important to clarify these issues in order to prevent and control the reproductive damages caused by n-hexane. Therefore, we conducted in vivo and in vitro experiments 
to investigate the impact of $n$-hexane on the secretion of ovarian estradiol and progesterone and carried out analysis and discussion.

From the in vivo and in vitro experiment results, we found the following. 1) In the in vivo experiments, after the inhalation of different doses of n-hexane, serum progesterone levels decreased significantly, which was statistically significant compared to the control group $(\mathrm{P}<$ 0.01); however, serum estradiol levels did not change. This indicates that n-hexane has an apparent reproductive endocrine interference function, especially on progesterone levels. 2) In both in vivo and in vitro experiments, after exposure to n-hexane or 2,5-HD, both estradiol and progesterone levels decreased, and there were statistical significant differences compared to the control groups. Compared to in vitro experiments, the results from in vivo experiments need to account for the balance and regulation of hormone secretion in the ovary by the hypothalamus-pituitary-ovarian axis. Therefore, the above results show that n-hexane directly inhibits the secretion of ovarian $\mathrm{E}_{2}$ and $\mathrm{P}_{4}$, and this inhibition cannot be compensated by in vivo hormone regulation mechanisms. 3 . At different stages of the estrous cycle (such as estrus and diestrus), the sensitivity of ovarian cells to toxic chemical materials is significantly different. In the current study, the effects of 2,5-HD on the secretion of estradiol and progesterone by ovarian cells were compared. Results show that at different stages of the estrous cycle, the secretion function of ovarian cells was significantly inhibited, which was statistically significant compared to the control groups $(\mathrm{P}<0.05)$; differences were especially obvious at the estrus and diestrus stages. In summary, n-hexane can inhibit ovary secretion of $\mathrm{E}_{2}$ and $\mathrm{P}_{4}$, the direct inhibition of ovary sex hormone secretion might be an important mechanism for its endocrine interference function.

\section{ACKNOWLEDGEMENTS}

Thanks for teachers Lin Wei and Wang Zhangjing was assisting in this study. Thanks for National Nature Science Foundation of China (30972514).

\section{REFERENCES}

[1] Wang, W.Y. and Wu, A.S. (2003) The investigation of Nhexane in Putian and Quanzhou city. Strait Journal of Preventive Medicine, 1, 31-32.

[2] Alan, H.D. and Lauren, N. (2006) Textbook of obstetrics \& gynecololgy. Science Publishing Company, New York.

[3] Sallmen, M., Neto, M. and Mayan, O.N. (2007) Reduced fertility among shoe manufacturing workers. Occupational and Environmental Medicine, 65, 518-524. doi:10.1136/oem.2007.032839

[4] Xu, X.C. (2004) Chronic n-hexane poising in clinic. Industrial Disease, 30, 175-177

[5] Moenter, S.M., DeFazio, A.R. and Pitts, G.R. (2003) Mechanisms underlying episodic gonadotropin-releasing hormone secretio. Front Neuroendocrinol, 24, 79-93. doi:10.1016/S0091-3022(03)00013-X

[6] Buffet, N.C. and Bouchard, P. (2001) The neuroendocrine regulation of the human ovarian cycle. Chronobiology International, 18, 893-919.

[7] Carelli, V., Franceschini, F. and Venturi S., (2007) Grand rounds: Could occupational exposure to n-hexane and other solvents precipitate visual failure in Leber here ditary optic neuropathy? Environmental Health Perspectives, 1, 113-115

[8] Sendur, O.F., Turan, Y., Bal, S. and Gurgan, A. (2009) Toxic neuropathy due to N-hexane: Report of three cases. Inhalation Toxicology, 21, 210-214. doi:10.1080/08958370802311169

[9] Sun, Y., Lin, Y., Li, H., Liu, J., Sheng, X. and Zhang, W. (2011) 2,5-Hexanedione induces human ovarian granulosa cell apoptosis through BCL-2, BAX, and CASPASE3 signaling pathways. Archives of Toxicology, in press. doi:10.1007/s00204-011-0745-7 